\title{
Do the Stripes on Three-Dimensional Models Overcome the Odor Signals in Tabanids Landing Choice?
}

\author{
Gabriela Vaduva \\ Department of Biology, Lund University, Lund 22362, Sweden \\ E-mail: vaduva_gabriela@yahoo.se
}

Received: August 31, 2020 Accepted: September 26, 2020

doi:10.5296/jbls.v11i2.17807 URL: https://doi.org/10.5296/jbls.v11i2.17807

\begin{abstract}
Information about the important factors in tabanid flies visual orientation to hosts has been largely derived from experimental modifications of visual traps and decoys. In the present study performed in wood pasture (Hästhult), southern Sweden, three-dimensional striped models resembling the shape of Zebra, Bongo, Kudu and four control models of different homogenous colors (black, white, reddish-brown and brown) were baited with acetone and aged cow urine in order to test the behavioral preferences in terms of visual and olfactory stimuli in host-seeking tabanids. Attraction of tabanid flies to these models (3D) was high, possibly due to the greater visibility from several directions and also from a greater distance. Vision is important in activating, orienting tabanid flies to the host, as well as for their decision whether and where to land. This research revealed that the visual cues such as stripes on striped models became increasingly important in directing tabanids landing and searching behavior at close range. Likewise, the tabanids approach to attractants sources was overridden by visual cues (stripes) at greater extent compared with the more attractiveness to homogenous colors on control models. Moreover, the visual stimuli (stripes) played also a supplementary role, modifying the selection of landing area on striped model (land on homogenous color part in Bongo and Kudu) once alighting responses were initiated by odor. Tabanid species, especially Haematopota pluvialis and Tabanus bromius exhibited a preference for landing mostly on reddish-brown control model when given the choice of other colors. However, the complex interaction of attractants and visual cues (stripes, color, shape) in the later stages of resource location, remains relatively little studied in all species of tabanids.
\end{abstract}

Keywords: African Mammals, Attractants, Attractiveness, Olfaction, Stripes, Tabanidae, Vision, Zebra 


\section{Introduction}

During the centuries, a lot of scientists tried to solve the mysteries with regard to animals. There are a variety of reasons to believe that every moment counts on important discovery. The color patterns in animals and their function, especially, white, black coats and also striped patterns found some explanations, but the mechanism by each pattern evolved is still unknown (Murray, 1981; Caro, 2009).

With regard to the question - why does the Zebra have stripes? there are some theories but still none of them is definite. One explanation suggests that the stripes have a camouflage effect, helping to break up the shape of animals for predators. However, the behavior of zebras, especially as they never attempt to hide but rest in groups in open areas, does not fit very well with this idea. Another explanation is based on the idea that stripes are creating an optical illusion which is avoided by predators, but escaping in zebras is possible mainly because of their speed and stamina (Morris, 1991). Yet another explanation could be that both black and white stripes dazzle the predator when it is in the close proximity of the Zebra. Finally, thermoregulation, social benefits, protection against tsetse flies have been suggested, and a new theory is based on the association between striping and temperature (Ruxton, 2002; Larison et al., 2015). Unfortunately, none of these theories has the right answer and the puzzle of the stripes on Zebra and other African Mammals pelages continues to confuse and fascinate. An interesting theory has recently attracted a lot of research: do the stripes on different African Mammals act as an evolutionary force by reducing attraction of tabanid flies? Nevertheless, the striping in African Mammals could be extremely complex and it is unwise to say that the pattern only appeared to avoid the tabanids attacks.

Apart from zebras, striped mammals are also found in some of the other mammal families such as Giraffidae and Bovidae. Carnivores also have a large number of different patterns including stripes, blotch, spots and so on (Vaduva, 2016).

In the family Bovidae, some species such as Greater Kudu (Tragelaphus strepsiceros), Lesser Kudu (Tragelaphus imberbis) and Bongo (Tragelaphus eurycerus) display from 9 to 15 vertical, thin, white stripes on the sides which can vary individually in number, spacing and boldness. In some animals, the white stripes are forked with bars and spots between them. Moreover, body color of these three species varies from cinnamon, grey-brown in Kudu to reddish-brown in Bongo (Kingdon, 1982).

The family Tabanidae is a common group of flies occurring worldwide in which the females feed on blood of mammals including humans. Males tabanids are strictly nectar feeders and in a few species, both sexes feed on nectar and pollen. The larvae of tabanids are terrestrial that are living mainly in litter and soil, aquatic and also semi-aquatic. In terms of the transmission of disease, a few health problems in humans could be associated with tabanids. Because, horse flies and deer flies are quickly feeders that visit the same host repeatedly, especially, when they are interrupting, give them the potential to move disease organisms from host to host such as tularemia and also anthrax. It is recognized that tabanid flies are not developing these pathogens inside them. However, there is a relationship between deer flies and the filarial worm causing Loa loa in central and western Africa. As a consequence of their blood feeding habits, 
tabanids cause considerably weight loss and lower milk production in cattle. When their number is high enough they could also be remarkably annoying (Lehane, 2005).

It was experimentally demonstrated, that tabanid flies are attracted to certain color hosts that emit greater polarization signals such as black surfaces comparing with white surfaces (Horváth et al., 2008). Likewise, it has been investigated that the introduction of striped patterns reduces attractiveness to tabanids. The results showed a strong repellency by tabanids in the same time with the introduction of stripes and least attraction to narrow zebra stripes. Simultaneously, other attraction experiment pointed out a significant repellency of tabanids by striped area of Okapi comparing with such great attractiveness to its homogenous black surface (Vaduva, 2016).

As a reason why striped patterns determine so high repellence to tabanid flies it has been suggested that they hinder polarotaxis (Horváth et al., 2008).

In any case, two interesting hypotheses gain terrain proposing the main function of zebra stripes is not to defeat polarotaxis by tabanid flies but to confuse these flies and also to conceal the Zebra from them (Britten et al., 2016).

In tabanids as well as in other insects, vision and olfaction play a complex role in the location of mates and other necessary resources. The importance of vision to tabanids, especially in pursuing the females by males is not very well studied. Vision could be markedly important in orientation, especially activation of tabanid species. The tabanids color preferences might determine their landing on different color decoys (Allan et al., 1987). According to Kelber (2001), insect eyes perceive colors as the human's eyes do, but with different sets of photoreceptors. Color choice behavior is based on the spectral types of photoreceptors. Results described in the present paper highlight the importance of understanding the entire picture of an interesting host-parasite system. More specifically, my purpose was to investigate how tabanid flies respond to striped patterns of different colors and also to homogenous colors baited with cattle aged urine and acetone. (i) Could the visual signals of striped patterns be overcome through olfactory signals used by tabanids in locating host animal? (ii) What role do the visual stimuli (stripes) play in the selection of the landing area on striped models, once alighting responses are initiated by airborne olfactory cues? (iii) Which host color is more attractive to every species of tabanids?

\section{Materials and Methods}

\subsection{Study Area}

The study was performed at a cattle farm in Hästhult (N 56 19' 12.58", E $13^{\circ} 33^{\prime} 32.85^{\prime \prime}$ ), southern Sweden between July 20 and August 31, 2016. All the observations were done in a large wood pasture surrounded by a mixed deciduous-coniferous forest, with a large pond in the close proximity. At a distance of approximately $50 \mathrm{~m}$, there was the farm with a huge stable where the cattle are housed. The total number of cattle was 125. During the whole period of study, some of the cows rested outside the stable, while others were grazing on broad areas, somewhat managing to surround two or three parts of the research site. 


\subsection{Tabanids Attraction Experiment}

Seven 3D models of different colors were used, trying to imitate as closely as possible the real shape of African mammals. They were built on wooden frames covered with Masonite mimicking the height of the animals and all the main parts of their shape. Over the Masonite, oilcloth of different colors was mounted. Four control models were uniformly: black, white, brown and reddish-brown (Figures b1-b4). The others three models representing Zebra, Bongo and Kudu were black, brown and reddish-brown painted with white paint over the oilcloth where there are the stripes of African Mammals. Since it was impossible to find a reddish-brown oilcloth close to the natural color of Bongo, a white oilcloth was painted with a suitable color (odorless, water based acrylic paint), resulting almost perfectly in that reddish-brown color of this animal (Figures a1-a3).

Each model was $140 \mathrm{~cm}$ long, $70 \mathrm{~cm}$ wide and $100 \mathrm{~cm}$ height (Figure 2). The size of the contact surfaces was $140 \mathrm{~cm} \times 100 \mathrm{~cm}(\mathrm{~L} \mathrm{x} \mathrm{H})$ on each side, resulting in a total size of the contact surface of each model of $140 \mathrm{~cm} \times 200 \mathrm{~cm}(\mathrm{~L} \mathrm{x} \mathrm{H})$. The height from the ground to the top of each model was $150 \mathrm{~cm}$. Both sides of each model were almost parallel imitating the shape of each animal and having the upper part imitating that upper side of the animal. Each model was fixed $20 \mathrm{~cm}$ deep in the ground. With regard to the black model with stripes (Zebra model), there were three orientations of the stripes: vertical, horizontal and oblique. The length of the surface occupied by the vertical stripes was $93 \mathrm{~cm}$ and the height $100 \mathrm{~cm}$, followed by the length of the horizontal stripes being $47 \mathrm{~cm}$ and the height $50 \mathrm{~cm}$. The area covered with oblique stripes had the same length and height as that with the horizontal stripes. The stripes had different widths depending on their orientation and color. As regards the vertical stripes, the average width of white stripes was $1.70 \mathrm{~cm}$ and the black ones $2.50 \mathrm{~cm}$. Likewise, for oblique stripes, the average width of white stripes was $2.4 \mathrm{~cm}$ and the black ones $3 \mathrm{~cm}$. Also, for horizontal stripes, the average width of white stripes was $1.2 \mathrm{~cm}$ and the black ones $1.4 \mathrm{~cm}$. In terms of the Bongo 3D model, the length of the unstriped surface ("neck") was $62 \mathrm{~cm}$ and the length of the striped surface $78 \mathrm{~cm}$ including 12 stripes of white color $(1.6 \mathrm{~cm})$ and reddish color $(4.9 \mathrm{~cm})$. Furthermore, on the Kudu 3D model, the length of the unstriped surface ("neck") was $26 \mathrm{~cm}$ and the length of the striped surface $114 \mathrm{~cm}$ including 12 stripes of white color $(1.1$ $\mathrm{cm})$ and brown color $(8.4 \mathrm{~cm})$. The number of stripes was the average number of stripes between Lesser Kudu and Great Kudu. In all 3D striped models, the chosen stripe widths pointed out the average for black and white stripes (for each part of body) from all specimens and also species within the same group (depending on stripe orientation) after the measurements in museums in three countries (Vaduva, 2016). All 3D models have been covered with Temmen insect glue, produced by TEMMEN GmbH Germany. The glue is non-toxic, odorless, colorless and weather-resistant. It was refreshed three times a week. The 3D models were set parallel to each other in a single row oriented N-S, with a sunny (S) and a shady $(\mathrm{N})$ side. The distance between models was $15 \mathrm{~m}$, while the distance to each of the two forest edges was $50 \mathrm{~m}$. Moreover, to the ecotone area was a distance of about $100 \mathrm{~m}$. All 3D models were baited with aged cow urine (aged about 2 weeks in order to develop the phenolic components) and acetone (as a long range orientation cue; purity 100\%). These attractants were placed in two different glass vials (500 $\mathrm{ml}$ aged cow urine and $100 \mathrm{ml}$ pure acetone) on 


\section{Macrothink

the ground inside each model. Both types of vapor were dispersed through different opening diameters, $0.4 \mathrm{~cm}$ for acetone and $4 \mathrm{~cm}$ for cow urine. Whenever needed, the vials with attractants were refilled with similarly aged urine and acetone.

Using an environment anemometer LUTRON LM-8000, the temperature was measured daily at $6 \mathrm{pm}$.

\subsection{Collecting and Processing Specimens}

All tabanid flies were collected daily between 19 and $20 \mathrm{~h}$. The specimens were placed in labeled plastic vials. When the time did not allow for species determination the same day, the vials were placed in a freezer at a temperature of minus $18^{\circ} \mathrm{C}$ overnight, and individuals were identified and counted the next day.

The tabanids were identified to species level according to descriptions of species and keys from Chvála et al., (1972); Walker (1994) and Oosterbroek (2006).

\subsection{Data Analysis}

In some of the analyses, the number of tabanid flies was transformed to $\log 10(n+1)$ before the computation of variance analyses, as regards the complying with the normal distribution.

The statistical analyses were performed using SPSS V22.0.

\subsection{Spectral Reflectance}

Reflections from reddish-brown, black, brown and white colors of 3D models were measured using a Maya 2000 Spectrometer (Ocean Optics) calibrated with a diffuse reflectance standard (WS1, Ocean Optics) under with a high-powered, continuous Xenon Light Source HPX-2000. Spectra were measured at $1 \mathrm{~nm}$ intervals from 300 to $700 \mathrm{~nm}$. All four spectra are displayed in (Figure 1a). The reflectances measured at $45^{\circ}$ and $90^{\circ}$ angle demonstrate how much light of which wavelengths is reflected diffusely (measured at the $45^{\circ}$ angle) and visible on cloudy days. The reflectance measured at $90^{\circ}$ angle is the sum of diffusely reflected and mirror-reflected light, which would be maximal on sunny days and which would be seen by a tabanid approaching the trap from the direction of the sun. The mirror-reflected light is white sunlight, and polarized (Figure 1b). 


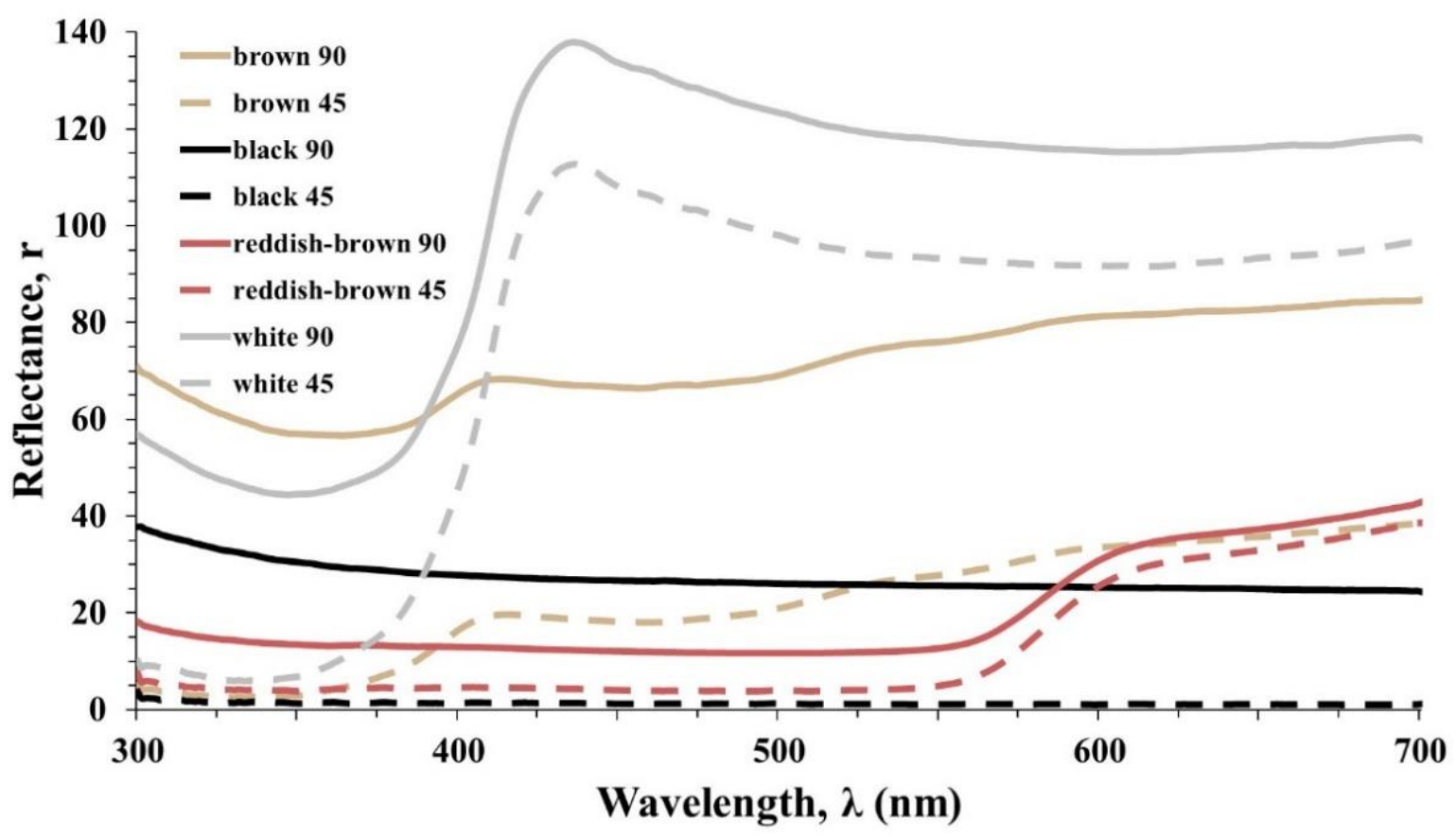

Figure 1a. Reflection spectra of all models colors. The reflectance curves at $90^{\circ}$ are represented by solid lines and at $45^{\circ}$ by dotted lines

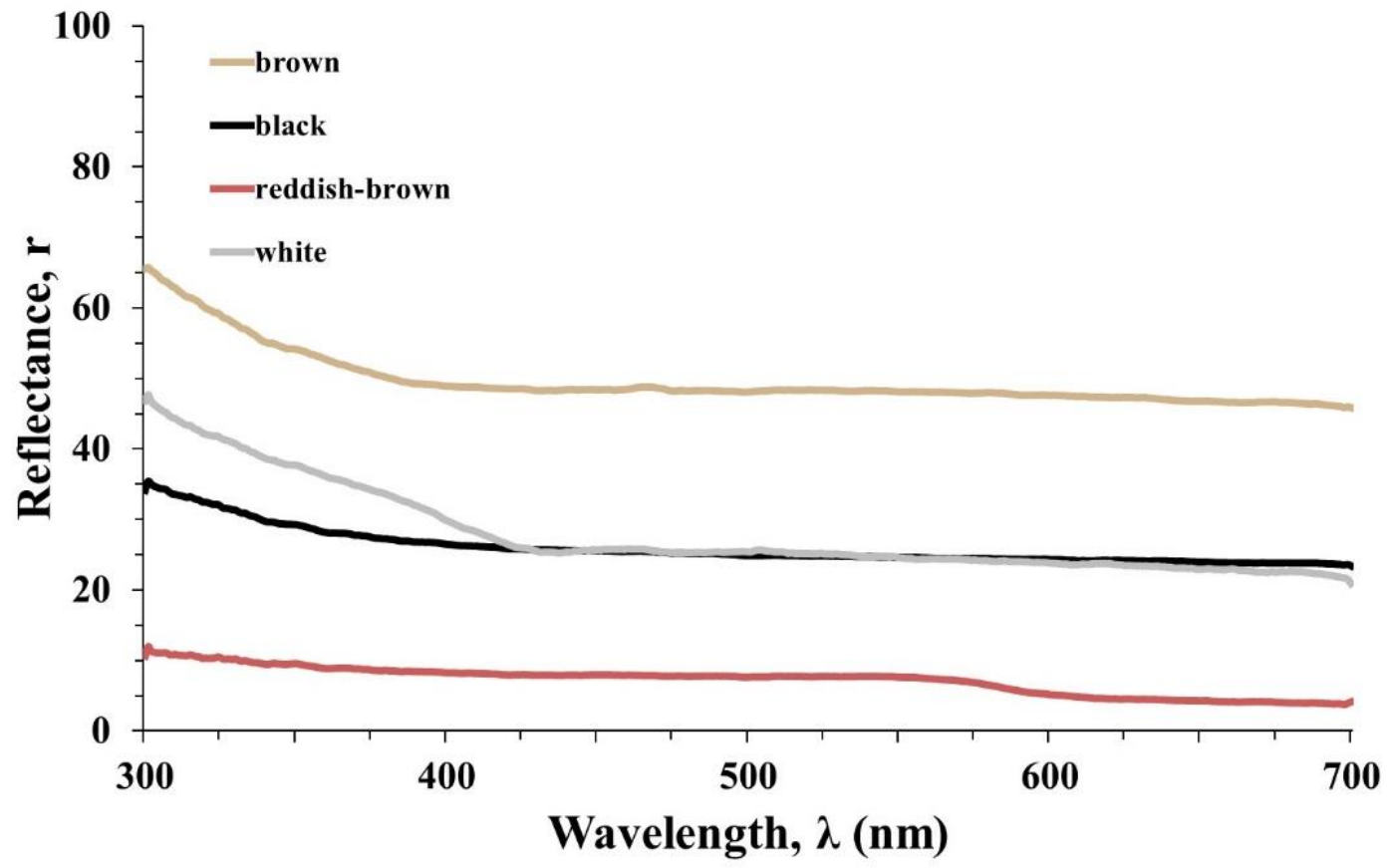

Figure 1b. Polarized white light reflection of the black, reddish-brown, brown and white 3D models 


\section{I Macrothink}
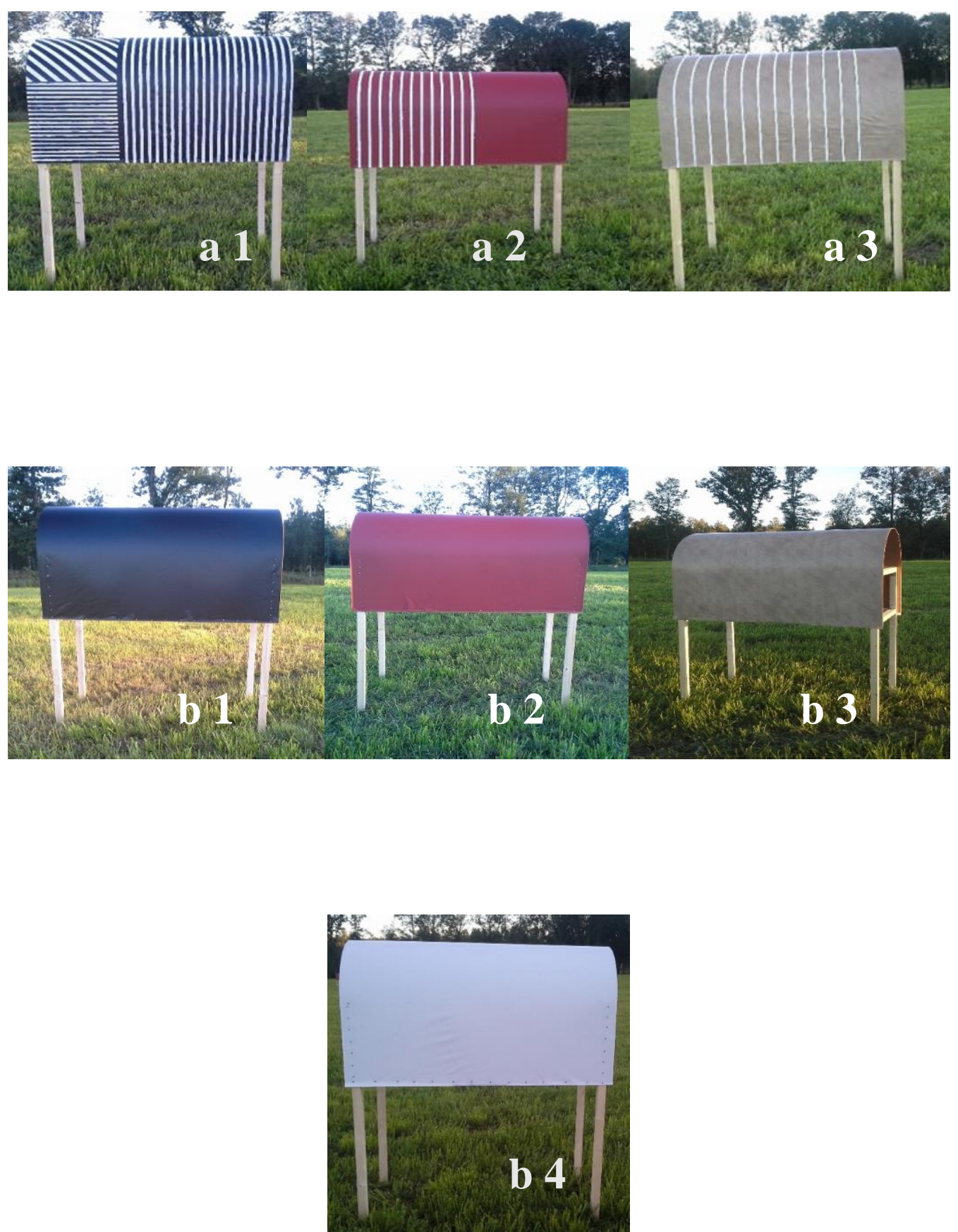

Figure 2. Three-dimensional baited models experiment performed in Hästhult, Sweden, 2016. Attractiveness of tabanids to Zebra, Bongo, Kudu models (a1-a3) and the four control models (black, reddish-brown, brown and white; b1-b4) 


\section{Results}

In this study, tabanid flies were captured in a total number of 4137 on all 3D models (Figures a1-a3; b1-b4). As regards the species, 15 species of Tabanidae (Diptera) were caught during the entire research period. The number of tabanids caught on the uniformly colored control models (Figures b1-b4) was high. The most abundantly collected species on these control models were Haematopota pluvialis $(\mathrm{n}=1003)$, Tabanus bromius $(\mathrm{n}=563)$, Tabanus maculicornis $(\mathrm{n}=425)$, Hybomitra ciureai $(\mathrm{n}=311)$, followed by Haematopota subcylindrica $(\mathrm{n}=295)$, Hybomitra muehlfeldi $(\mathrm{n}=199)$ and Tabanus glaucopis $(\mathrm{n}=186)$ (Table 1). Moreover, the number of tabanids specimens caught on 3D striped models (Figures a1-a3) was much lower comparing with capturing rate of tabanids on control models. The most abundant species being the same as those captured on homogeneous colors but with a significantly lower number (Haematopota pluvialis, $\mathrm{n}=166$; Tabanus bromius, $\mathrm{n}=59$; Tabanus maculicornis, $\mathrm{n}=44$; Hybomitra ciureai, $\mathrm{n}=31$ and Tabanus glaucopis, $\mathrm{n}=16$ ) (Table 2).

Table 1. Tabanids species collected on 3D control models

\begin{tabular}{|c|c|c|c|c|}
\hline Species & $\begin{array}{l}\text { Black } \\
\text { control } \\
\text { model }\end{array}$ & $\begin{array}{c}\text { Bongo } \\
\text { control } \\
\text { model }\end{array}$ & $\begin{array}{l}\text { Kudu } \\
\text { control } \\
\text { model }\end{array}$ & $\begin{array}{l}\text { White } \\
\text { control } \\
\text { model }\end{array}$ \\
\hline Chrysops caecutiens L., 1758 & 39 & 51 & 29 & 26 \\
\hline Chrysops divaricatus Loew, 1858 & 27 & 26 & 20 & 16 \\
\hline Hybomitra bimaculata Macquart, 1826 & 34 & 38 & 30 & 19 \\
\hline Hybomitra ciureai Séguy, 1937 & 104 & 88 & 63 & 56 \\
\hline Hybomitra distinguenda Verrall, 1909 & 6 & 13 & 10 & 5 \\
\hline Hybomitra kaurii Chvála et Lyneborg, 1970 & 27 & 38 & 25 & 14 \\
\hline Hybomitra lundbecki Lyneborg, 1959 & 11 & 27 & 26 & 21 \\
\hline Hybomitra muehlfeldi Brauer, 1880 & 53 & 68 & 46 & 32 \\
\hline Haematopota pluvialis L., 1758 & 259 & 354 & 219 & 171 \\
\hline Haematopota subcylindrica Pandellé, 1883 & 118 & 89 & 45 & 43 \\
\hline Heptatoma pellucens Fabricius, 1776 & 19 & 43 & 30 & 43 \\
\hline Tabanus bovinus L., 1758 & 4 & 9 & 4 & 1 \\
\hline Tabanus bromius L., 1758 & 149 & 198 & 111 & 105 \\
\hline Tabanus glaucopis Meigen, 1820 & 28 & 58 & 52 & 48 \\
\hline Tabanus maculicornis Zetterstedt, 1842 & 114 & 146 & 84 & 81 \\
\hline TOTAL & 992 & 1246 & 794 & 681 \\
\hline
\end{tabular}

Notes: All values indicate the attraction of each species of tabanids to the four different colors of the control surfaces (b1-b4). The collection period has been recorded from July 20 to

August 31, 2016. 
Table 2. Tabanids species collected on striped 3D models

\begin{tabular}{lrrr}
\hline \multicolumn{1}{c}{ Species } & $\begin{array}{r}\text { Zebra } \\
\text { model }\end{array}$ & $\begin{array}{r}\text { Bongo } \\
\text { model }\end{array}$ & $\begin{array}{c}\text { Kudu } \\
\text { model }\end{array}$ \\
\hline Chrysops caecutiens L., 1758 & 0 & 13 & 11 \\
\hline Chrysops divaricatus Loew, 1858 & 0 & 3 & 5 \\
\hline Hybomitra bimaculata Macquart, 1826 & 0 & 4 & 6 \\
\hline Hybomitra ciureai Séguy, 1937 & 1 & 16 & 14 \\
\hline Hybomitra distinguenda Verrall, 1909 & 0 & 1 & 1 \\
\hline Hybomitra kaurii Chvála et Lyneborg, 1970 & 0 & 7 & 4 \\
\hline Hybomitra lundbecki Lyneborg, 1959 & 0 & 9 & 7 \\
\hline Hybomitra muehlfeldi Brauer, 1880 & 0 & 8 & 7 \\
\hline Haematopota pluvialis L., 1758 & 6 & 103 & 57 \\
\hline Haematopota subcylindrica Pandellé, 1883 & 0 & 4 & 2 \\
\hline Heptatoma pellucens Fabricius, 1776 & 0 & 11 & 5 \\
\hline Tabanus bovinus L., 1758 & 0 & 0 & 0 \\
\hline Tabanus bromius L., 1758 & 3 & 33 & 23 \\
\hline Tabanus glaucopis Meigen, 1820 & 0 & 8 & 8 \\
\hline Tabanus maculicornis Zetterstedt, 1842 & 0 & 23 & 21 \\
\hline \multicolumn{1}{c}{ TOTAL } & 10 & 243 & 171 \\
\hline
\end{tabular}

Notes: All values indicate the attraction of each species of tabanids to the striped patterns of Zebra, Bongo and Kudu models (a1-a3). Collection period was recorded from July 20 to August 31, 2016.

There was a statistically significant difference between Zebra, Bongo and Kudu 3D models in the number of captured tabanid flies (ANOVA: $F_{2,126}=31.864, P<0.001$ ). The difference in number of tabanids caught on Bongo model and Zebra model was highly significant (Tukey HSD, $P<0.001)$ as was the difference in number between Kudu model and Zebra model (Tukey HSD, $P<0.001$; Figure 3).

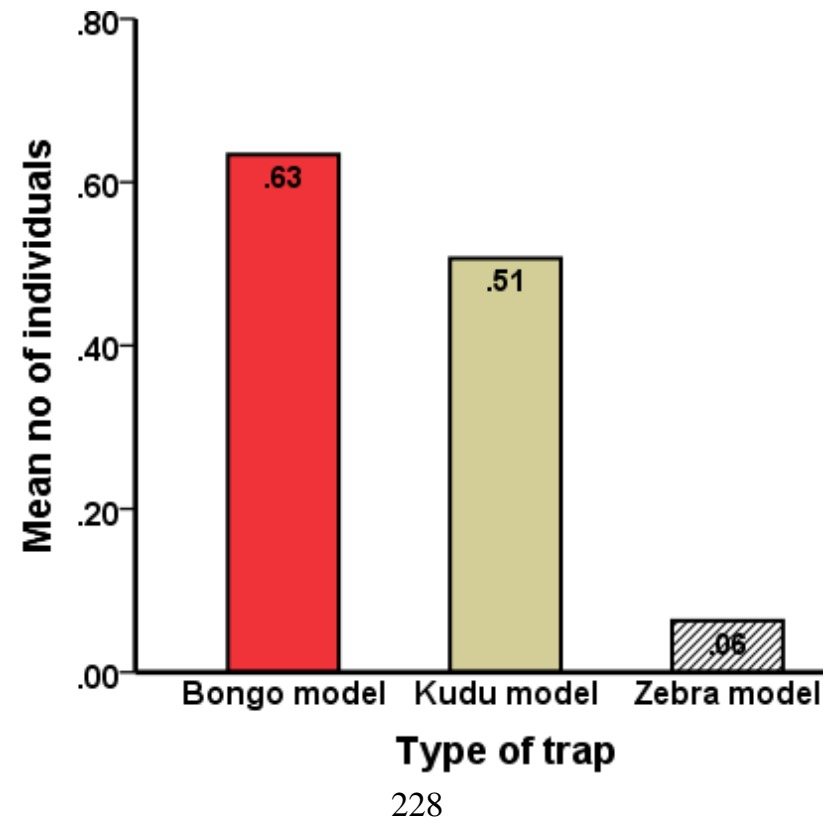




\section{Macrothink}

Figure 3. Relation between Bongo, Kudu and Zebra 3D models in terms of attractiveness to tabanid flies. All 3D models (a1-a3) have the same dimensions. The widths of stripes displayed the averages of black and white stripes from all specimens measured on animals' skin in the museums according with my published data (2016)

The difference in the number of tabanid flies caught on black, respectively white 3D control models and Zebra 3D model with oblique, horizontal and vertical stripes orientation was statistically significant (ANOVA: $F_{2,126}=59.443, P<0.001$ ). Tukey HSD also revealed highly significant difference in tabanids mean numbers between Zebra 3D model and both 3D black and white control models $(P<0.001$; Figure 4$)$.

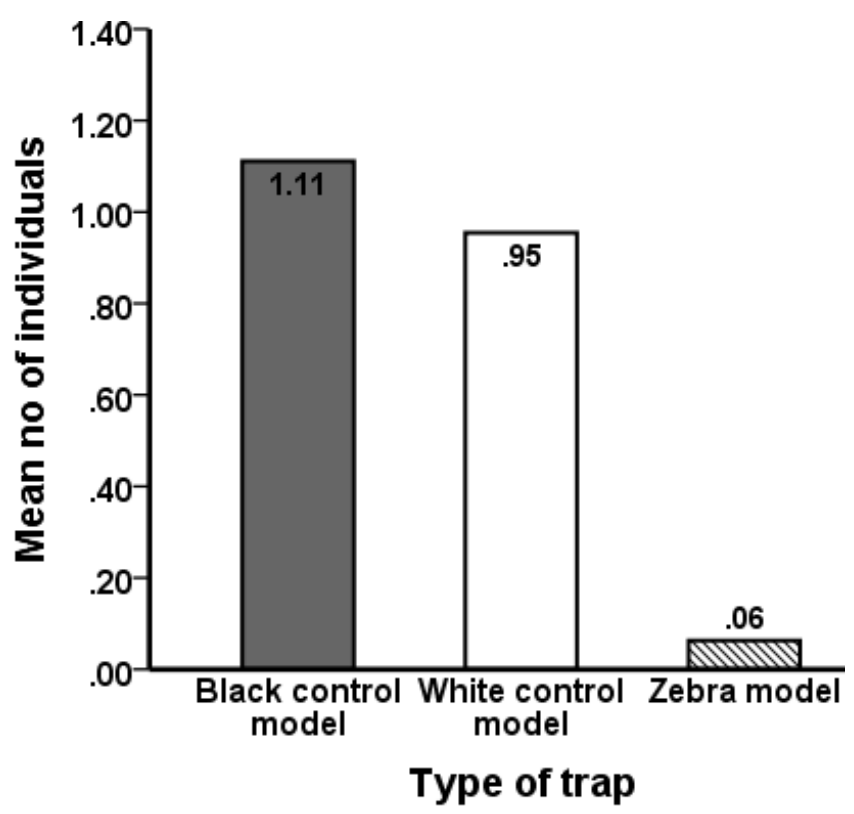

Figure 4. The number of tabanids caught on 3D black and white control models and Zebra 3D model the entire period

The difference in capture tabanid flies on Bongo 3D control model, Bongo 3D model, Bongo 3D model unstriped surface and striped surface was statistically significant (ANOVA: $\left.F_{3,168}=58.401, P<0.001\right)$. Tukey HSD showed highly significant differences in number of tabanids caught on Bongo control model and all the other types of surfaces $(P<0.001)$, respectively Bongo model and Bongo model striped area $(P<0.001)$ and also between Bongo model unstriped area and Bongo model striped area $(P<0.001$; Figure 5$)$. 


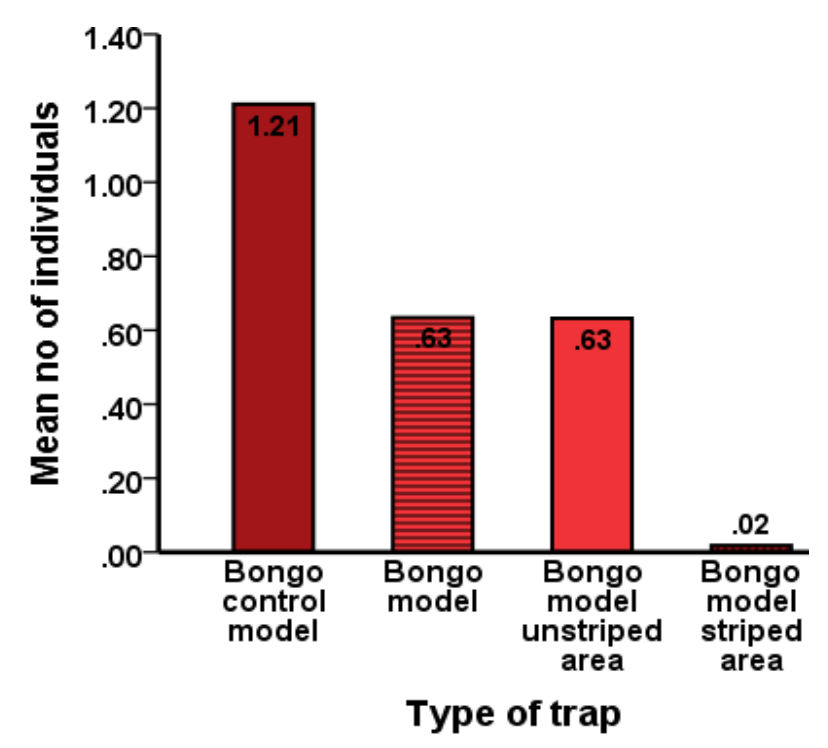

Figure 5. Number of tabanids caught on Bongo 3D control model (b2) and Bongo 3D model (a2), as well as on the unstriped and striped area of the Bongo 3D model. The influence of stripes on the Bongo model is underlined by the smaller attraction of flies and, especially to the unstriped area

Likewise, there was a statistically significant difference in captured tabanid flies on Kudu 3D control model, Kudu 3D model, Kudu 3D model unstriped surface and striped surface (ANOVA: $F 3,168=46.435, P<0.001$ ). Tukey HSD revealed a significant difference in the number of tabanids between Kudu control model and all the other types of surfaces $(P<0.001)$, respectively Kudu model and Kudu model striped area $(P<0.001)$ and also between Kudu model unstriped area and Kudu model striped area $(P<0.001$; Figure 6$)$.

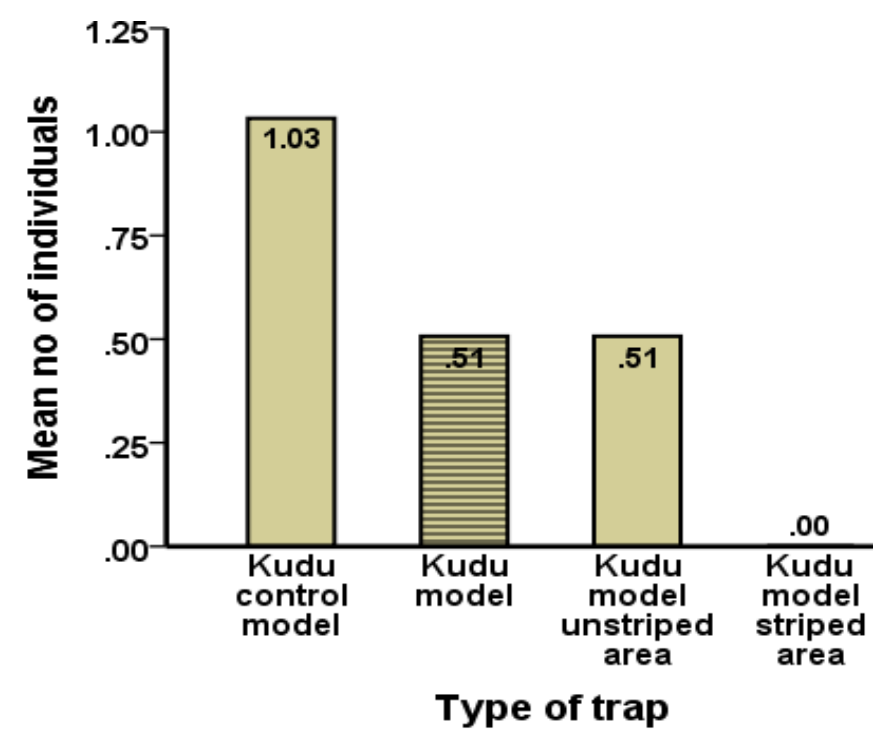

Figure 6. The relationship between Kudu 3D control model (b3) and Kudu 3D model (a3), respectively Kudu 3D model unstriped area and striped area as regards the attractiveness to tabanids. The influence of stripes insertion is signaled by the attraction of a smaller number of flies and, especially on the stripless side 


\section{Macrothink}

Between all 3D control models (Black, Bongo, Kudu, White) there was a statistically significant difference (ANOVA: $F$ 3,168 $=3.056, P=0.030$ ) in terms of mean number of captured tabanids. Tukey HSD pointed out a significant difference regarding the average number of caught tabanids, only between Bongo control model and White control model $(P=0.027 ;$ Figure 7$)$.

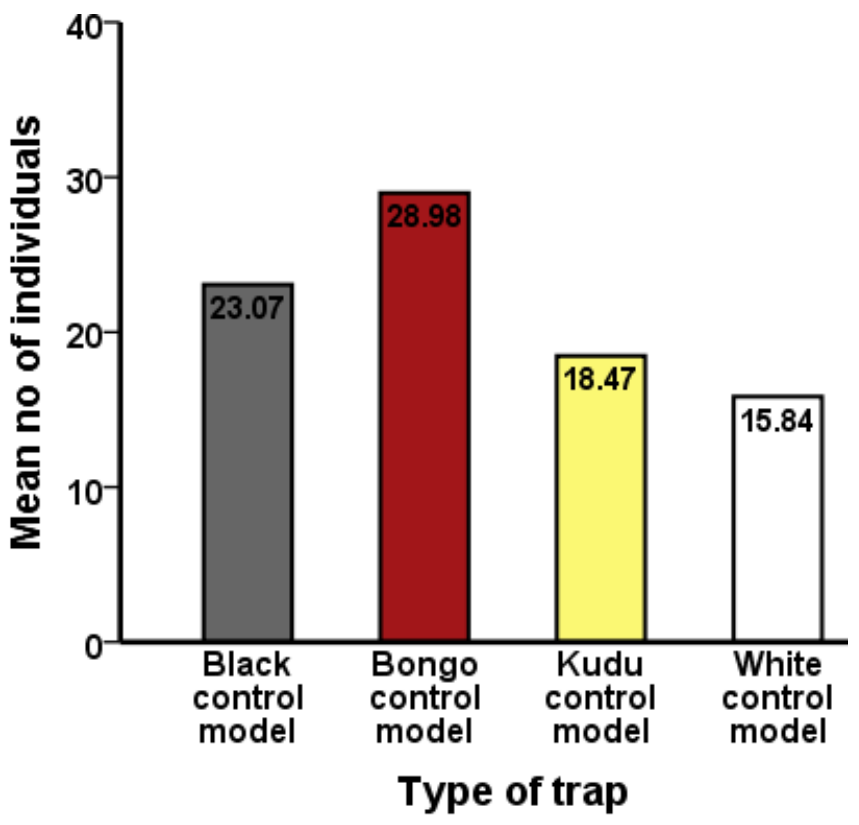

Figure 7. Relation between all 3D control models (b1-b4) (black, Bongo-reddish, Kudu-brown and white) in terms of tabanids attractiveness to four different colors. The surface area of all 3D control models has the same dimensions

When the differences in mean number of captured tabanid flies between the sunny, and shady vertical sides, and the upper side of the black control model were compared (Figure 8a), for Bongo control model (Figure 8b), Kudu control model (Figure 8c) and white control model (Figure 8d), the results showed a highly significantly differences (black control model: ANOVA: $F{ }_{2,126}=10.622, P<0.001$, Tukey HSD: sunny side and shady side, $P=0.01$; sunny side and upper side, $P<0.001$; Bongo control model: ANOVA: $F$ 2,126 $=8.579$, $P<0.001$, Tukey HSD: sunny side and upper side, $P<0.001$; Kudu control model: ANOVA: $F$ 2,126 $=9.552, P<0.001$, Tukey HSD: sunny side and upper side, $P<0.001$; white control model: ANOVA: $F$ 2,126 $=10.241, P<0.001$, Tukey HSD: sunny side and upper side, $P<0.001$; Figures $8 \mathrm{a}, \mathrm{b}, \mathrm{c}, \mathrm{d})$. 
(a)

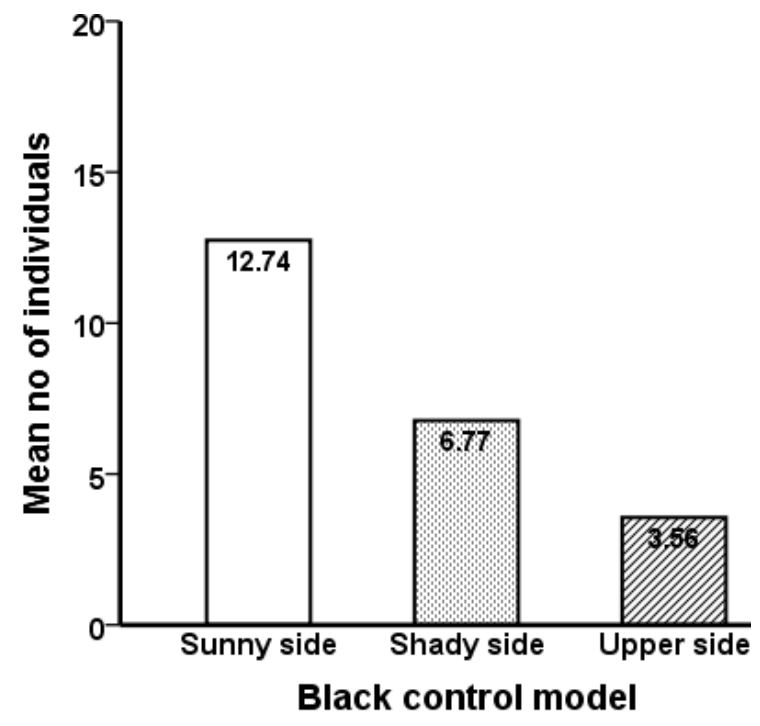

(c)

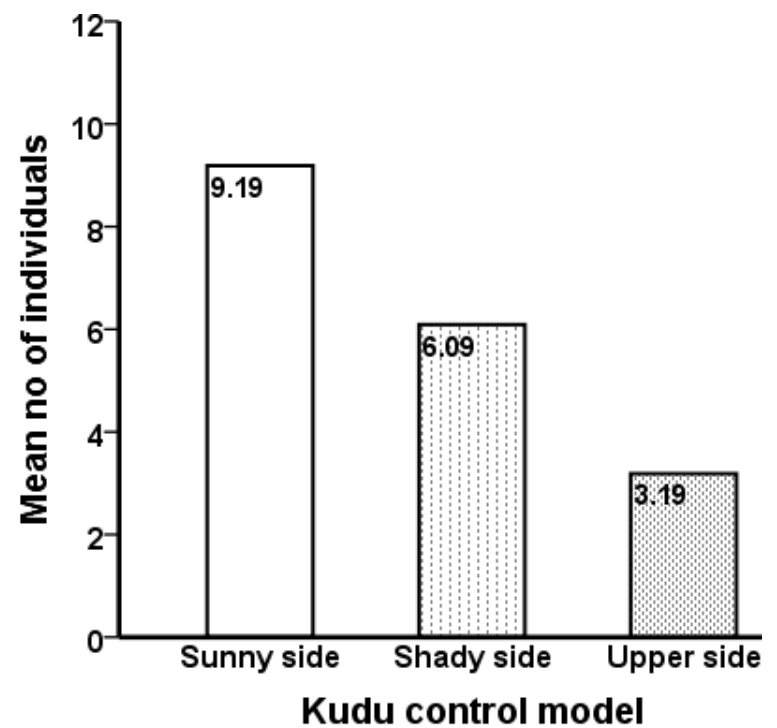

(b)

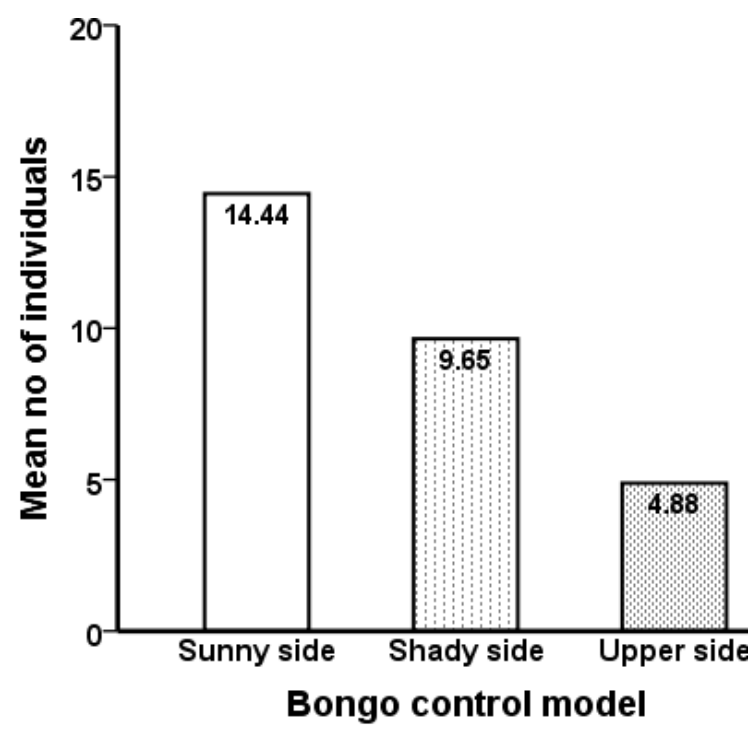

(d)

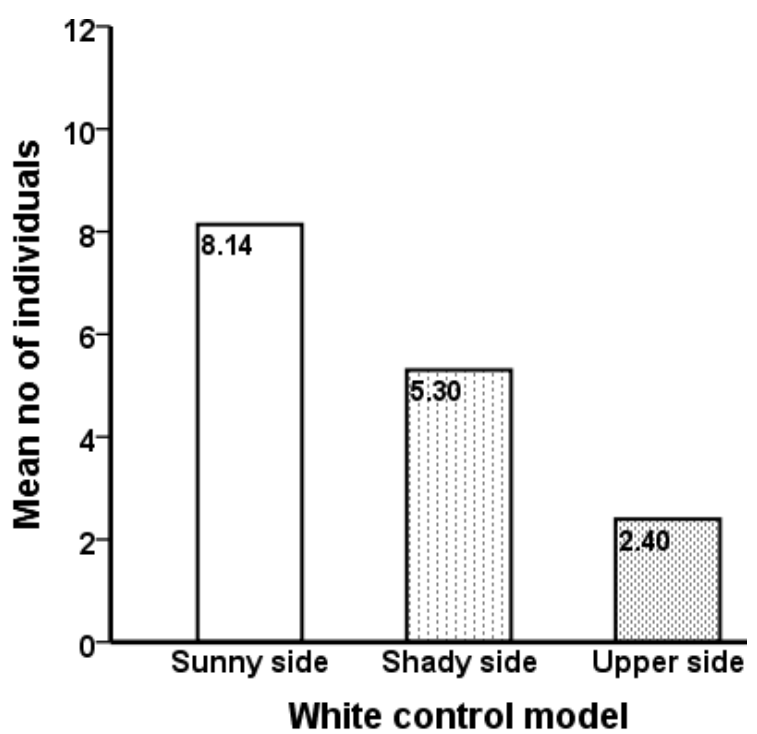

Figures 8. The attractiveness of tabanids to the three sides of all 3D control models (black, Bongo, Kudu and white). Comparison of mean tabanids number captured on sunny, shady and upper side of (a) black control model, (b) Bongo control model, (c) Kudu control model and (d) white control model

The number of tabanid flies captured on all types of weather (sunny, partly cloudy, cloudy and rainy) during the experimental period differed significantly (ANOVA: $F$ 3,39 $=22.711$, $P<0.001)$. Tukey HSD showed significant differences in the total number of tabanids captured on sunny vs. cloudy weather $(P<0.001)$; sunny vs. rainy weather $(P<0.001)$; partly cloudy vs. rainy weather $(P<0.001)$; cloudy vs. rainy weather $(P=0.001$; Figure 9$)$. 


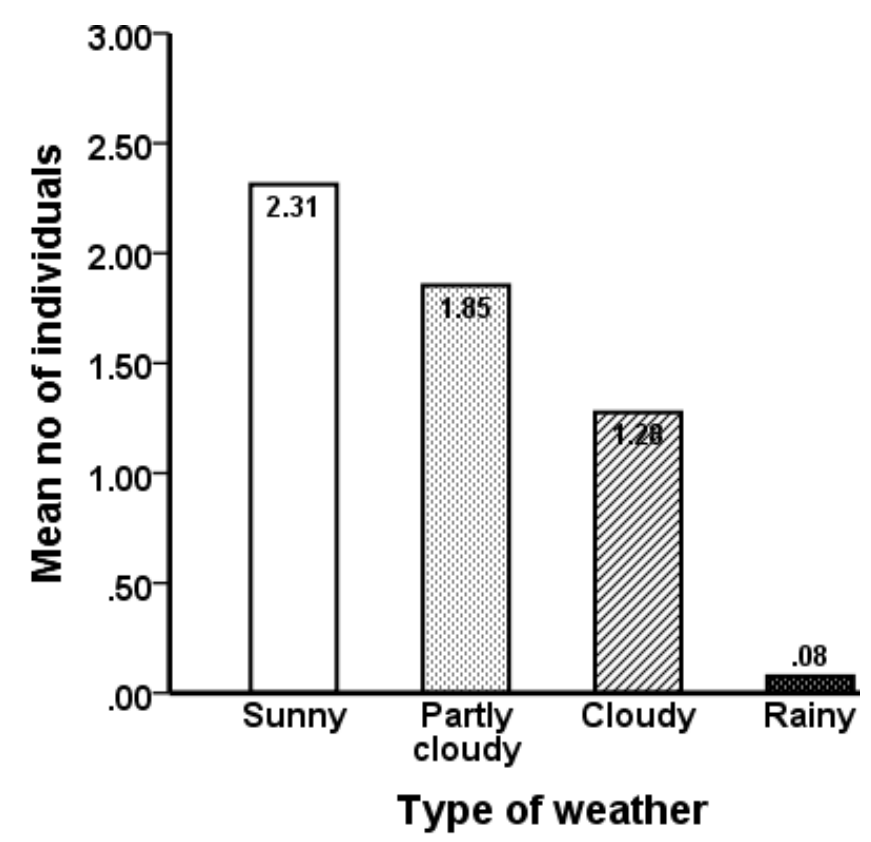

Figure 9. The relationship between tabanids capture rate and all types of weather. Above the columns are displayed the mean numbers of all captured tabanid flies depending on different type of weather

With regard to tabanid species attractiveness to different color of control models there was a statistically significant difference between all of them (ANOVA: $F_{3,2576}=8.486, P<0.001$ ). Tukey HSD revealed differences in the mean number of tabanids only between Bongo control model and Kudu control model $(P=0.001)$ and, respectively Bongo control model and White control model $(P<0.001$; Figure 10$)$. 


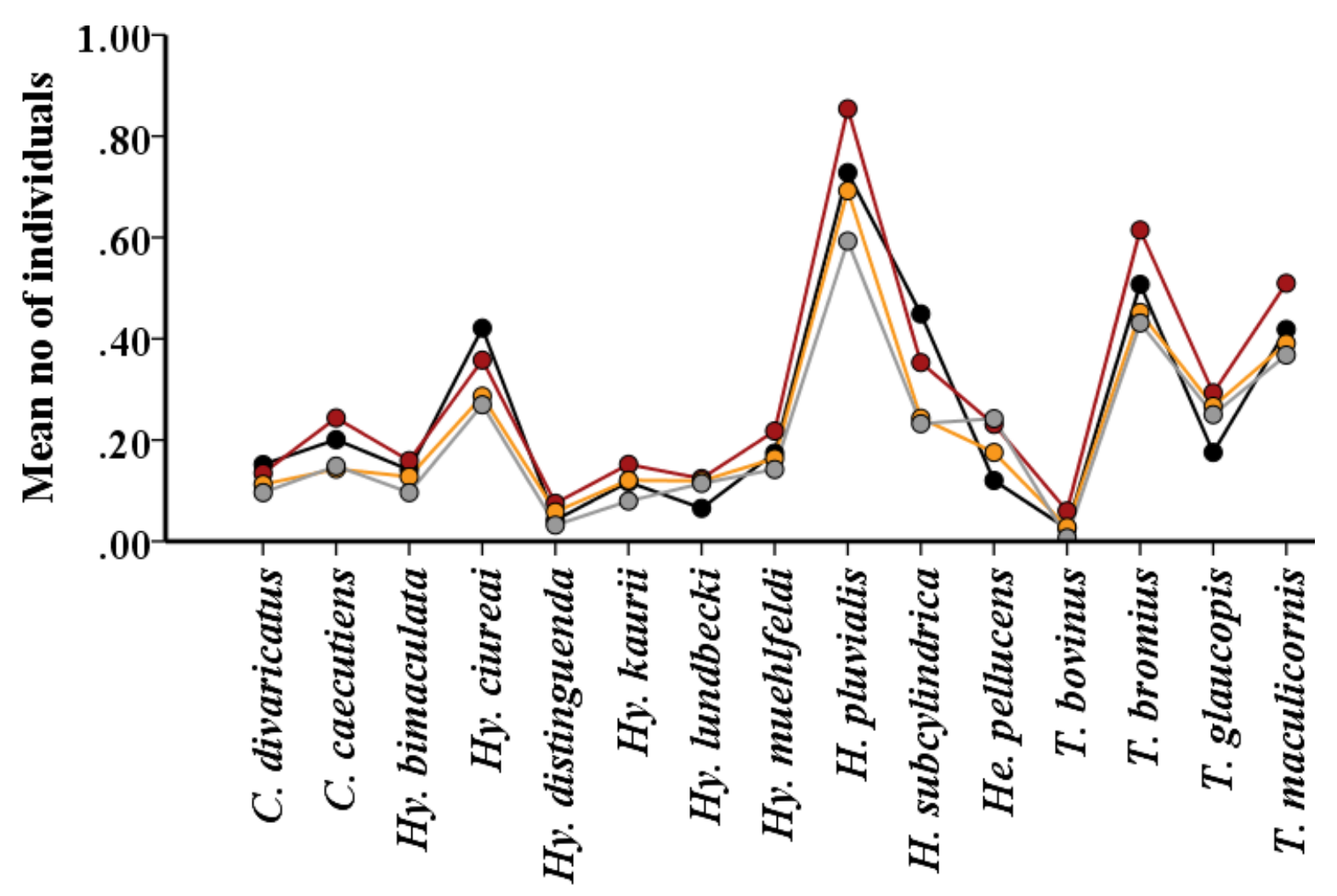

Tabanid species

Figure 10. Attractiveness of tabanids species to different types of color (reddish-brown, brown, black and white) of all 3D control models. Mean number of each species of tabanids caught on these homogenous colors

\section{Discussion}

\section{Tabanids attraction experiment}

This study revealed for the first time that not just the stripes of Zebra are repellent for tabanids, but also the stripes of two other large mammals, the Bongo and Kudu, although at a lower efficiency. Perhaps, because the stripes do not cover the entire body in these African mammals in comparison with Zebra.

Observations that stripes themselves are visible from a distance of more than 20 meters compared to the polarization contrast between stripes, support the hypothesis that any striped pattern according to Vaduva (2016) is repellent to tabanids, for a totally independent reason of polarotaxis (Britten et al., 2016).

The overall results show that the tabanid flies obviously evade all striped surfaces to a lesser or greater extent. One explanation could be that the stripes might conceal the Zebra, also possibly Kudu and Bongo, at moderate distances from tabanids, and the other explanation consist in confusing their landing behavior at close range. When the tabanids pass from olfactory to visual signal between five to ten meters, the optics of their eyes would 
considerably blur the signals of white and black stripes (Larison, 2014; Britten et al., 2016). It could be also possible that the number, orientation and width of stripes play an important role in the number of tabanids landings on striped models. In such a way one might explain the small number of tabanids (10) collected from the Zebra 3D model compared to the Bongo (243) and Kudu (171) 3D models. One hypothesis could be that on these models the stripes do not cover the entire body and do not have the same width as Zebra stripes. Moreover, the stripes of Zebra with a small width were markedly repellent to tabanids and no specimen landed on this type of stripes.

Similarly, the approach of tabanid species to sources of odorants (such as acetone and aged cow urine) was overridden by visual cues such as stripes at close range, determining the flies to orientate towards the unstriped part of models. Orientation in tabanids has been shown to be remarkably dependent on olfactory stimuli and landing behavior on visual cues such as color. More likely, the complex interaction of odors and visual cues in terms of resource location remains not entirely studied in tabanids. An improved understanding of such interactions may allow to develop more efficient and effective traps in order to enhance the control of these flies.

Tabanids landed in the presence of odor and thus the strong visual cues provided by stripes on Zebra, Bongo or Kudu models made them to orientate their landing mainly on homogenous colors (reddish, brown, black and white). This result indicates that although odor cues enhanced the induction of tabanids landing, visual cues such as stripes acted as a strong repellent in the selection of a final landing site. Normally, tabanid flies are activated by host odor and then by smell follow that host from a distance. When it will be in the close range of the host, intervene the visual contact with all visual information that will be used in the orientation final stages (Lehane, 2005). In terms of the used attractants, the combination of acetone and aged cow urine played a significant role in tabanid flies attraction to homogenous 3D control models (b1-b4). Their number differed on these models, but the explanation could be based on tabanids color choice as well as on the insect's ecology. Host-seeking tabanids respond greatly to such as color blue, red and black and also white for some species (Allan $e t$ $a l .$, 1987). My findings in this work pointed out a high number of tabanids captured on reddish, black, brown and white homogenous models colors. Likewise, there was no significant difference in capturing rate between black and white colors.

According to Sasaki (2001), a red-absorbing visual pigment was never documented in tabanids flies. In this scenario, the red / reddish color may appear as dark gray or even black for tabanids. The contrast also plays a primary role in the visual attraction of the tabanids to decoys, respectively dark and saturated blue, providing the maximum contrast against a background of grass.

With respect to tabanids attractiveness, the three-dimensional models were extremely efficient. Probably due to the fact that they are visible from many directions and even from a great distance and also because they resembled quite well the shape of the animal. The visual attraction of tabanids decreased when the uniform colors were interrupted by stripes. Clearly, 3D models caught more tabanids than two-dimensional decoys (Allan et al., 1987). 


\section{MlMacrothink}

Journal of Biology and Life Science

ISSN 2157-6076

2020, Vol. 11, No. 2

Tabanid flies are active during the day, in a warm weather and most of the species are prevalent only from June to August thus being present simultaneously. In my work, most catches of tabanids were done on sunny days with an average temperature of $23.9^{\circ} \mathrm{C}$ and also on partly cloudy days when the average temperature was $19^{\circ} \mathrm{C}$. Between both types of weather there was no significant difference due to the high number of Haematopota pluvialis captured also on overcast conditions because this species needs to keep its body surface permanently hydrated. In terms of tabanids capture rate on sunny, shady and upper sides in all control models, the result carries out a significant difference only between sunny and upper side for all models. However, the black control model has signaled a significant difference not only between sunny and upper side but also between sunny and shady side in number of captured tabanids. The result was not surprising, as it has been experimentally demonstrated earlier that the capture rate of the tabanids was higher on the sunny side compared to the shady side of black patterns (Vaduva, 2016).

Tabanid flies are attracted to specific shapes, but not all species are equally attracted. Some species are not at all attracted by certain shapes. They revealed species-specific differences in host location strategies (Allan et al., 1987). Regarding to my study, the species of tabanids pointed out the significant differences in the attractiveness of control models, especially between Bongo and Kudu control models, and also between Bongo and white control models. The reddish-brown control model (Bongo) was the most attractive to almost each species of tabanids, especially to the species which were caught in higher numbers such as Haematopota pluvialis, Tabanus bromius, Tabanus maculicornis, Hybomitra ciureai followed by Haematopota subcylindrica, Hybomitra muehlfeldi and Tabanus glaucopis. The Bongo control model was followed in the species attractiveness by the black control model, that in particular attracted the same species as the reddish color model. A very similar behavioral pattern of tabanid species, was found for the last two control models (Kudu and white). This result reveals that there are preferences for each of tabanids in choosing the host color. Furthermore, the preference has been maintained throughout the entire period of study. Moreover, certain species of tabanids may be attracted to the same color of the host more than the other colors. In general, the preferred color for tabanids landing is the one involved in their activation and orientation (Lehane, 2005).

The relation between the number of tabanids on the 3D control models and the light reflectance at $90^{\circ}$ appears to be inversely proportional. Thus, the lower the reflectance, the higher the number of tabanids. A model with a lower reflectance may be more visible against the bright background, especially on sunny days. Thus, such a model may be easier to detect by the simple visual systems of the tabanid flies.

Interestingly, this relation is preserved for light reflectance at $45^{\circ}$, with the exception of black and bongo control models. Although, the reddish-brown color has a somewhat higher reflectance than black, the differences are rather small for the wavelengths at which the tabanids are photopositive. This relation may also be explained by the fact that generally, the response of host-seeking tabanids is greatest to blue, red and black, and only for some species to white. 
No similar relation was found in regards to the amount of polarized light and the control models. The Bongo control model had the lowest amount of polarized light and the highest number of tabanids. Nevertheless, the white model which had the least number of tabanids, has a lower reflectance of polarized light compared with the Kudu control model, and similar or identical for most of the wavelengths with the black model.

Certainly, further investigations might be important in order to understand the tabanids species-specific differences in host location strategies.

\section{Acknowledgments}

I thank Anders Svensson for his agreement to use his land and for providing me with cattle aged urine and also Denis Vaduva for field assistance. I would like to thank Berne Svensson and Ann - Catherine Svensson for their hospitality and agreement to build all the 3D models on the land of their company and Michael Vaduva for his help in building and installing them in the field. I express my deeply gratitude to Professor Almut Kelber for valuable comments that improved a previous version of the manuscript and together with Peter Olsson for their invaluable aid with the spectrophotometry measurements. I am grateful for the valuable feedback from the others two anonymous readers of the manuscript. This research work was supported by a grant from "Entomologiska Sällskapet i Lund" (to G. V.) and I would like to thank Mikael Sörensson in this respect.

\section{References}

Allan, S. A., Day, J. F., \& Edman, J. D. (1987). Visual Ecology of biting flies. Annual Review of Entomology, 32, 297-316. https://doi.org/10.1146/annurev.en.32.010187.001501

Britten, K. H., Thatcher, T. D., \& Caro, T. (2016). Zebras and Biting Flies: Quantitative Analysis of Reflected Light from Zebra Coats in Their Natural Habitat. PLOS ONE, 11, e0154504. https://doi.org/10.1371/journal.pone.0154504

Caro, T. (2009). Contrasting coloration in terrestrial mammals. Philosophical Transaction of the Royal Society B, 364, 537-548. https://doi.org/10.1098/rstb.2008.0221

Chvála, M., Lyneborg, L., \& Moucha, J. (1972). The horse flies of Europe (Diptera, Tabanidae). Copenhagen: Entomological Society of Copenhagen

Hanec, W., \& Bracken, G. K. (1962). Responses of female horse flies (Tabanidae: Diptera) to light. Annals of the Entomological Society of America, 55, 720-721. https://doi.org/10.1093/aesa/55.6.720

Horváth, G., Majer, J., Horváth, L., Szivák, I., \& Kriska, G. (2008). Ventral polarization vision in tabanids: horseflies and deerflies (Diptera: Tabanidae) are attracted to horizontally $\begin{array}{llll}\text { polarized light. } & \text { Naturwissenschaften, } & \text { 95, }\end{array}$ https://doi.org/10.1007/s00114-008-0425-5

Kelber, A. (2001). Receptor based models for spontaneous colour choices in flies and butterflies. Entomologia Experimentalis et Applicata, 99, 231-244. https://doi.org/10.1046/j.1570-7458.2001.00822.x 


\section{Macrothink Institute $^{\mathrm{TM}}$}

Kingdon, J. (1982). East African Mammals. An Atlas of Evolution in Africa. vol IIIC, Bovids. London: University of Chicago Press edition 1989. London: Academic Press (Inc.) London Ltd

Krčmar, S., Mikuska, A., \& Merdić, E. (2006). Response of Tabanidae (Diptera) to different natural attractants. Journal of Vector Ecology, 31, 262-265. https://doi.org/10.3376/1081-1710(2006)31[262:ROTDTD]2.0.CO;2

Larison, B., Harrigan, R. J., Thomassen, H. A., Rubenstein, D. I., Chan-Golston, A. M., Li, E., $\&$ Smith, T. B. (2015). How the zebra got its stripes: a problem with too many solutions. Royal Society Open Science, 2, 140452. https://doi.org/10.1098/rsos.140452

Lehane, M. J. (2005). The Biology of Blood-Sucking in Insects ( $2^{\text {nd }}$ edition). Cambridge: Cambridge University Press. https://doi.org/10.1017/CBO9780511610493

Morris, D. (1991). Animal watching: A field guide to animal behaviour. London: Arrow Books Limited

Murray, J. D. (1981). A pre-pattern formation mechanism for animal coat markings. Journal of Theoretical Biology, 88, 161-199. https://doi.org/10.1016/0022-5193(81)90334-9

Oosterbroek, P. (2006). The European Families of the Diptera Identification, diagnosis, biology. Utrecht: KNNV Publishing. https://doi.org/10.1163/9789004278066

Ruxton, G. D. (2002). The possible fitness benefits of striped coat coloration for zebra. Mammal Review, 32, 237-244. https://doi.org/10.1046/j.1365-2907.2002.00108.x

Sasaki, H. (2001). Comparison of capturing tabanid flies (Diptera: Tabanidae) by five different color traps in the fields. Applied Entomology and Zoology, 36, 515-519. https://doi.org/10.1303/aez.2001.515

Vaduva, G. (2016). The evolution of striped coat patterns in African mammals-what function do the stripes have? Journal of Biology and Life Science, 7, 53-70. https://doi.org/10.5296/jbls.v7i1.8613

Walker, A. (1994). Arthropods of humans and domestic animals. A guide to preliminary identification. London: Chapman \& Hall

\section{Copyright Disclaimer}

Copyright for this article is retained by the author(s), with first publication rights granted to the journal.

This is an open-access article distributed under the terms and conditions of the Creative Commons Attribution license (http://creativecommons.org/licenses/by/4.0/). 